\title{
The Yamada Polynomial of Spacial Graphs and Knit Algebras *
}

\author{
Jun Murakami \\ Department of Mathematics, Osaka University, Toyonaka, Osaka 560, Japan, and \\ Institute for Advanced Study, Princeton, NJ 08540, USA
}

Received April 20, 1992; in revised form January 15, 1993

\begin{abstract}
The Yamada polynomial for embeddings of graphs is widely generalized by using knit semigroups and polytangles. To construct and investigate them, we use a diagrammatic method combined with the theory of algebras $H_{N, M}(a, q)$, which are quotients of knit semigroups and are generalizations of Iwahori-Hecke algebras $H_{n}(q)$. Our invariants are versions of Turaev-Reshetikhin's invariants for ribbon graphs, but our construction is more specific and computable.
\end{abstract}

\section{Introduction}

In [Y], Yamada introduced an invariant $Y$ of embeddings of a spacial graph in $S^{3}$, which we call the Yamada polynomial. It is also an invariant of embeddings of a trivalent graph in $S^{3}$. It is one of the simplest cases of the invariants for ribbon graphs in [R-T], which are constructed by using a triangular Hopf algebra. Here, we generalize the Yamada polynomial from a different point of view. We first give a two-variable extension $Z_{s}$ of the Yamada polynomial $Y$ by a naive way. Our invariant is related to the HOMFLY polynomial while the Yamada polynomial is related to the Jones polynomial. This extension $Z_{s}$ has further generalizations. We define them by using representation theory of knit semigroups, which is an extension of the braid groups. The edges are colored by irreducible representations of knit semigroups and the vertices are colored by diagrams on polyhedrons. These extensions are closely related to the invariants in $[\mathrm{R}-\mathrm{T}]$. For those invariants, vertices are colored by elements of certain vector spaces and our coloring corresponds to specify such elements actually. We mainly discuss invariants related to the HOMFLY polynomial, but we may apply our method to other link invariants.

By using Kauffman's bracket polynomial $\langle\cdot\rangle$, we can reconstruct the invariant $Y$. The bracket polynomial is a version of the Jones polynomial [Jo], and is a regular isotopy invariant of non-oriented link diagrams defined by a relation

$$
\left\langle L_{x}\right\rangle=A\left\langle L_{0}\right\rangle+A^{-1}\left\langle L_{\infty}\right\rangle,
$$

\footnotetext{
* This research was supported in part by NSF grant DMS-9100383
} 
where $A$ is a non-zero complex parameter and $L_{x}, L_{0}, L_{\infty}$ are link diagrams that are identical except within a ball as in Fig. 1. We assume that $\langle\bigcirc\rangle=1$ for the trivial knot $\bigcirc$.

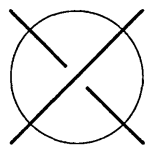

$\mathrm{L}_{\mathrm{x}}$

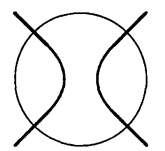

$\mathrm{L}_{0}$

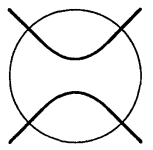

$\mathrm{L}_{\infty}$

Fig. 1

For a non-oriented spacial graph $\Gamma$, let $\mathscr{V}_{\Gamma}$ and $\mathscr{E}_{\Gamma}$ denote the set of its vertices and edges respectively. Let $2^{\Gamma}$ be the sets of all spacial graphs obtained by removing some (or no) edges from $\Gamma$. Let $G$ be a diagram of an embedding of $\Gamma$. As in Fig. 2, we get a link diagram $G^{(2)}$ from $G$. For $\Gamma^{\prime} \in 2^{\Gamma}$, let $G_{\Gamma^{\prime}}$ be the subdiagram of $G$ corresponding to $\Gamma^{\prime}$. Then $Y(G)$ is given by

$$
Y(G)=-\frac{A^{2}+A^{-2}}{A^{4}+1+A^{-4}} \sum_{\Gamma^{\prime} \in 2^{\Gamma}}\left(A^{2}+A^{-2}\right)^{\left|\mathscr{E}_{\Gamma^{\prime}}\right|-\left|\mathscr{E}_{\Gamma}\right|}\left\langle G_{\Gamma^{\prime}}^{(2)}\right\rangle
$$

where $\left|\mathscr{E}_{\Gamma^{\prime}}\right|$ and $\left|\mathscr{E}_{\Gamma}\right|$ denote the numbers of elements of $\mathscr{E}_{\Gamma^{\prime}}$ and $\mathscr{E}_{\Gamma}$. It is an invariant of embeddings of $\Gamma$ up to multiples of monomials in $A$. We can generalize the above construction in the cases of the Kauffman and HOMFLY polynomials. Jaeger did the Kauffman polynomial case in [Ja] and we do the HOMFLY polynomial case in this paper.

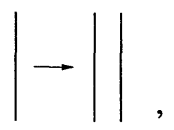

Fig. 2

edge

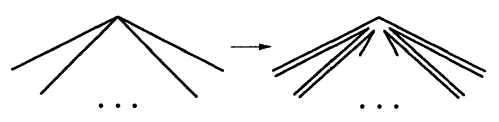

vertex

Let $P$ be the HOMFLY polynomial in [FY-H-LM-O]. It is an invariant of equivalence of oriented links and is a two-variable extension of the Jones polynomial. It is defined by the skein relation

$$
a^{-1} P\left(L_{+}\right)-a P\left(L_{-}\right)=\left(q-q^{-1}\right) P\left(L_{0}\right),
$$

where $a$ and $q$ are non-zero complex parameters not equal to 0 nor \pm 1 , and $L_{+}, L_{-}, L_{0}$ are link diagrams that are identical except within a ball as in Fig. 3. We assume that $P(\bigcirc)=1$ for the trivial knot $\bigcirc$.

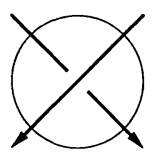

$\mathrm{L}_{+}$

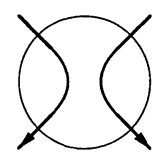

$\mathrm{L}_{0}$

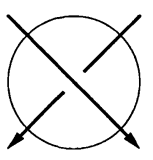

L.

Let $G$ be a diagram of an embedding of a non-oriented spacial graph $\Gamma$ and $s$ be a mapping from $\mathscr{V}_{\Gamma}$ to $\{ \pm 1\}$. Replacing the vertices and the edges as in Fig. 4, we 
get a linear combination of $2^{p} 2^{q}$ link diagrams, where $p$ and $q$ are the numbers of edges and vertices of $\Gamma$ respectively. Let $Z_{s}(G)$ be the HOMFLY polynomial of the above linear combination of diagrams. Then $Z_{s}(G)$ is an invariant of equivalence of embeddings of the graph $\Gamma$ up to a sign and multiplication by an integer power of $a$ (Theorem 2.3).

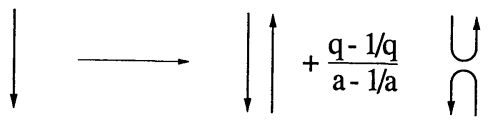

at an edge $\mathrm{e}$

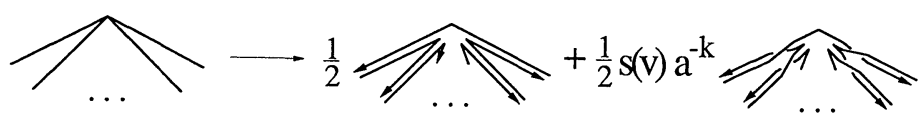

Fig. 4

at a vertex $\mathrm{v}$

On the other hand, Turaev-Reshetikhin introduced invariants of embeddings of a ribbon graph in [R-T] parametrized as follows. Let $\mathscr{A}$ be a triangular Hopf algebra. Fix a simple $\mathscr{A}$ module $V_{e}$ for each edge $e$ of $\Gamma$. For $v \in \mathscr{V}_{\Gamma}$, let $e_{1}, e_{2}, \ldots, e_{r}$ be the edges with end point $v$, End $_{v}=$ End $_{, b}\left(V_{e_{1}}^{\prime} \otimes V_{e_{2}}^{\prime} \otimes \ldots \otimes V_{e_{r}}^{\prime}, V_{0}\right)$, where $V_{0}$ is the trivial $\mathscr{t}$ module, $V_{e_{2}}^{\prime}=V_{e_{2}}$ if $v$ is the starting point of $e_{i}$ and $V_{e_{2}}^{\prime}=V_{e_{i}}^{*}$ (the contragradient module of $V_{e_{i}}$ ) if $v$ is the end point of $e_{i}$. Fix an element $I_{v} \in$ End $_{v}$ for every $v \in \mathscr{T}_{\Gamma}$. Then $\left(\mathscr{b} ; V_{e_{1}}, V_{e_{2}}, \ldots ; I_{v_{1}}, I_{v_{2}}, \ldots\right)$ parametrizes Reshetikhin-Turaev's invariants, where $\left\{e_{1}, e_{2}, \ldots\right\}=\mathscr{E}_{\Gamma}$ and $\left\{v_{1}, v_{2}, \ldots\right\}=\mathscr{D}_{\Gamma}$. The Yamada polynomial corresponds to the case $\mathscr{A}=\mathscr{U}_{q}\left(s l_{2}\right)$ and $V_{e}=V^{(3)}$ for any edge $e$, where $V^{(3)}$ is the 3-dimensional simple $\mathscr{C}_{q}\left(s l_{2}\right)$-module. in this case, $V^{(3)} \cong\left(V^{(3)}\right)^{*}$, and $I_{v}$ is unique up to a scalar multiple since $\operatorname{dim}\left(\right.$ End $\left.\mathscr{C}_{1}\left(V^{(3)} \otimes V^{(3)} \otimes V^{(3)}, V_{0}\right)\right)=1$. In this case, $V_{0}$ is the one-dimensional representation of $\mathscr{W}_{q}\left(s l_{2}\right)$. One problem remained in the work of [R-T]. They do not give a method to describe the element $I_{v}$. If $\operatorname{dim}\left(\operatorname{End}_{v}\right)=1$ then $I_{v}$ is unique up to a scalar multiple and so there is no problem. However, if $\operatorname{dim}\left(\operatorname{End}_{v}\right)>1$, we have to specify $I_{v}$.

In this paper, we construct similar invariants by using knit semigroups and we give colors of vertices by using a "polytangle," which is something like a tangle on a polyhedron. Our invariants are ambient isotopy invariant up to some scalar multiples. In the case of trivalent graphs, the category of embeddings of spacial graphs and that of abstract graphs are equivalent. Therefore, ours are invariants for embeddings of abstract trivalent graphs.

In Sect. 1, we investigate the two-variable extension $Z_{s}$ of the Yamada polynomial. In Sect. 2, we generalize $Z_{s}$ by using a polytangle, semigroup $B_{N, M}$ and an algebra $H_{N, M}(a, q)$. This algebra is a quotient of $\mathbf{C} B_{N, M}$ by the skein relation (1.3) and so a generalization of the Iwahori-Hecke algebra $H_{N}(q)$. In Sect. 3, we discuss some special cases, including the invariant in Sect. 1. To do this, we use representation theory of $H_{N, M}(a, q)$ developed in [Ko-M1].

\section{A Two-Variable Extension of the Yamada Polynomial}

2.1. Embeddings of a Spacial Graph. A spacial graph $\Gamma$ is an oriented or non-oriented graph with additional information. For each vertex $v \in \mathscr{T}_{\Gamma}$, we associate a disk $D_{v}$ 
and give an injective mapping from edges with end point $v$ to radii of $D_{v}$. For example, the graphs $\Gamma$ and $\Gamma^{\prime}$ in Fig. 5 are equivalent as abstract graphs, but are not equivalent as spacial graphs. If $\Gamma$ is oriented (resp. non-oriented) as a graph, then we call it an oriented (resp. non-oriented) spacial graph. Let $\Gamma$ be a spacial graph. An embedding of $\Gamma$ in $S^{3}$ is a set of mappings $f_{e}, f_{v}$, and $f_{D_{v}}$, where $f_{e}: e \rightarrow S^{3}$ for an edge $e, f_{v}: v \rightarrow S^{3}$ and $f_{D_{v}}: D_{v} \rightarrow S^{3}$ for a vertex $v$. We assume that these mappings are compatible with the additional information. Let $e$ be an edge with an end point at a vertex $v$. Then $f_{e}$ maps a neighborhood of $v$ in $e$ to $f_{D_{v}}(r)$, where $r$ is the corresponding radius of $e$. Let $G_{1}$ and $G_{2}$ be two embeddings of a spacial graph $\Gamma$. We say that $G_{1}$ and $G_{2}$ are equivalent if there is an isotopy of $S^{3}$ deforming $G_{1}$ to $G_{2}$.
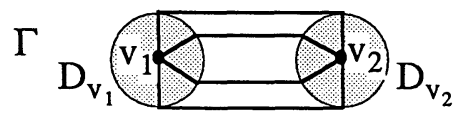

$\Gamma^{\prime}$

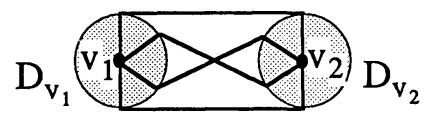

Fig. 5

As in the case of links, we define a diagram of a regular projection of an embedding $G$ of $\Gamma$. Regularity means that the projection has no triple point, and no double point at any vertex. Two diagrams $G_{1}$ and $G_{2}$ present equivalent embeddings if and only if there is a sequence of moves (RI)-(RV) in Fig. 6. Two diagrams $G_{1}$ and $G_{2}$ are called regular isotopic if there is a sequence of moves (RII), R(III), and (RIV) in Fig. 6.

(RI)

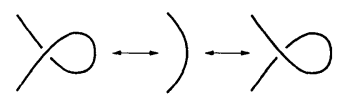

(RII)

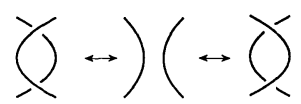

(RIII)

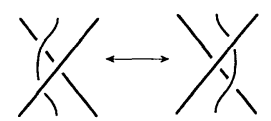

(RIV)

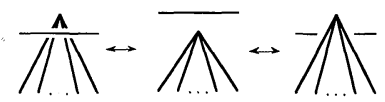

Fig. 6

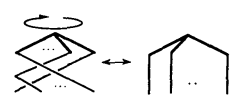

2.2. A Two-Variable Extension. Let $G$ be a diagram of an embedding of a non-oriented spacial graph $\Gamma$ and $s$ be a mapping from $\mathscr{T}_{\Gamma}$ to $\{ \pm 1\}$. We show that $Z_{s}$ in the introduction is an invariant of embeddings of $\Gamma$. By the definition of $Z_{s}$, it is a 
regular isotopy invariant of embeddings and it satisfies the following relations:par

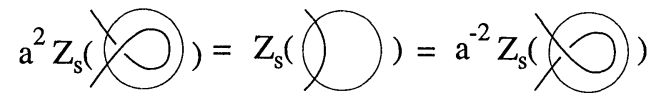

(ZV)

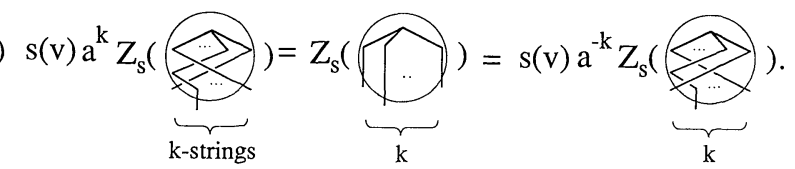

The diagrams of each relation are identical except within a ball. These relations are a consequence of the following relation, which comes from the skein relation (1.3):

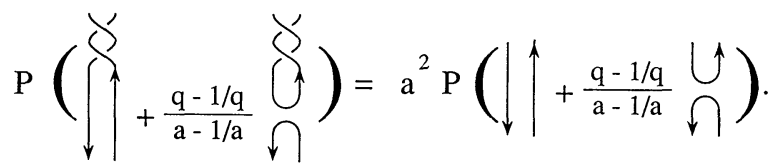

The relations (ZI) and (ZV) imply the following.

2.3. Theorem. The regular isotopy invariant $Z_{s}$ is an invariant of euivalence of embeddings up to a sign and multiplication by an integer power of $a$.

2.4. Another Two-Variable Extension. The Kauffman polynomial in [Ka2] of links is another two-variable extension of the Jones polynomial. Let $D$ be a regular isotopy invariant of non-oriented link diagrams defined by the following relations,

$$
\begin{gathered}
D\left(L_{+}\right)-D\left(L_{-}\right)=\left(q-q^{-1}\right)\left(D\left(L_{0}\right)-D\left(L_{\infty}\right)\right), \\
a^{-1} D\left(L_{p}\right)=D\left(L_{\mid}\right)=a D\left(L_{m}\right),
\end{gathered}
$$

where $a$ and $q$ are complex parameters with $a \neq \pm 1, q \neq \pm 1, a \neq \pm q$ and the link diagrams $L_{+}, L_{-}, \ldots$ are given in Fig. 7. The link diagrams in each relation are identical except within a ball. We assume that $D(\bigcirc)=1$ for the trivial knot $\bigcirc$. We call $D$ the $D$-polynomial. For an oriented link diagram $L$, let $F(L)=a^{-w(L)} D(|L|)$, where $|L|$ is the diagram $L$ without orientation and $w(L)$ is the writhe (sum of signatures of all the crossing points of $L$ ). Then $F$ is an invariant of link equivalence.
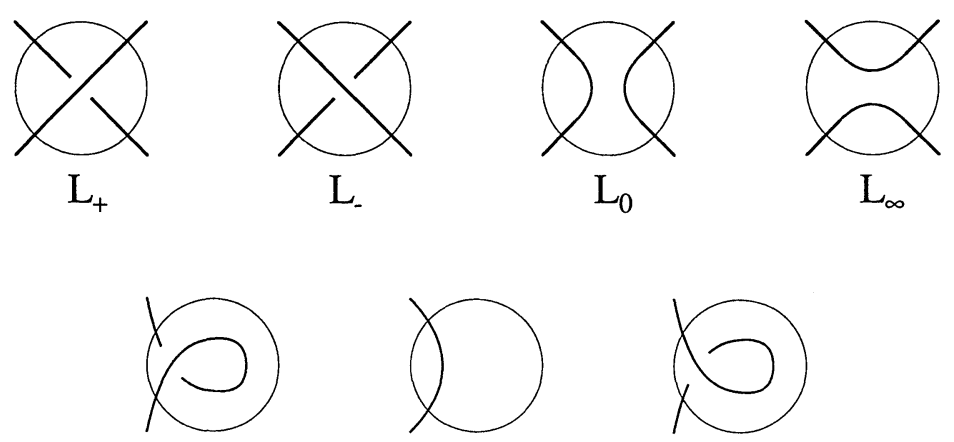

Fig. 7

$\mathrm{L}_{\mathrm{p}}$

$\mathrm{L}_{1}$

$\mathrm{L}_{\mathrm{m}}$ 
In [Ja], Jaeger defined an invariant of embeddings of non-oriented spacial graphs by using the invariant $D$. We naturally extend the $D$-polynomial to a linear combination of link diagrams. Let $G$ be a diagram of a non-oriented spacial graph $\Gamma$ and $s$ a mapping from the set of edges of $\Gamma$ to $\{ \pm 1\}$. Replacing the vertices and the edges as in Fig. 8, we get a linear combination of $3^{e}$ link diagrams, where $e$ is the number of edges of $\Gamma$. Let $J(G)$ be the $D$-polynomial of the above linear combination of diagrams. By its definition, $J$ is a regular isotopy invariant and it satisfies the following relations:

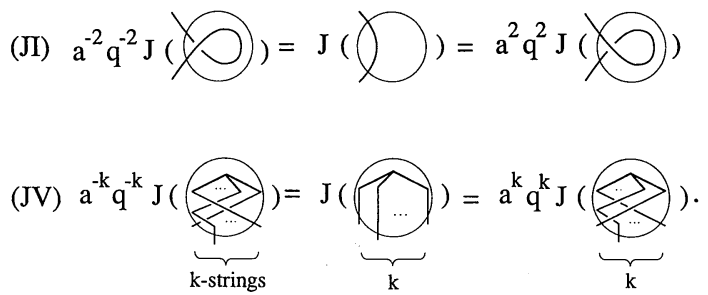

Therefore $J$ is an invariant of equivalence of embeddings up to a sign and multiplication by integer powers of $a q$.

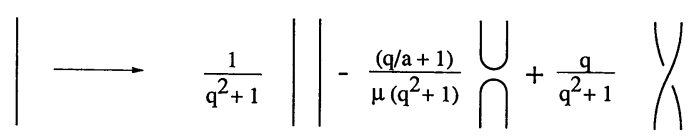

at an edge where $\mu=1+\frac{a-1 / a}{q-1 / q}$

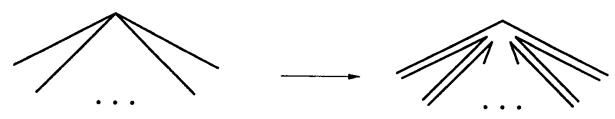

Fig. 8

at a vertex

2.5. Relation among Invariants. Since the $D$-polynomial and the HOMFLY polynomial are both two-variable extensions of the Jones polynomial, the invariants $Z_{s}$ and $J$ are a two-variable extension of the Yamada polynomial $Y$.

2.6. Theorem. For an embedding $G$ of a non-oriented spacial graph $\Gamma$, we have the following:

(1) $Y(G)=\left.\prod_{v \in \mathscr{T} \Gamma} 2\left(1+s(v)\left(-A^{3}\right)^{n_{v}}\right)^{-1} Z_{s}(G)\right|_{a=-A^{-4}, q=A^{2}}$, where $n_{v}$ is the degree
of $v$.

(2) $Y(G)=\left.J(G)\right|_{a=-A^{3}, q=A}$.

\section{Invariants of Embeddings of a Spacial Graph}

In this section, we assume that the parameters $a$ and $q$ in the definition of the HOMFLY polynomial is generic

3.1. Knit Semigroup. We first generalize the braid groups. Let $B_{N, M}^{\prime}$ be the free semigroup generated by $\left(\sigma_{1}^{+}\right)^{ \pm 1},\left(\sigma_{2}^{+}\right)^{ \pm 1}, \ldots,\left(\sigma_{N-1}^{+}\right)^{ \pm 1},\left(\sigma_{1}^{-}\right)^{ \pm 1},\left(\sigma_{2}^{-}\right)^{ \pm 1}, \ldots,\left(\sigma_{M-1}^{-}\right)^{ \pm 1}$, 
$\tau$, and $e$. These are corresponding to the diagrams in Fig. 9. As in the case of braids, the product of two elements $b_{1}$ and $b_{2}$ corresponds to the join of their diagrams. Two elements in $B_{N, M}^{\prime}$ are called equivalent if the corresponding diagrams are regular isotopic. The product structure of $B_{N, M}^{\prime}$ is compatible with this equivalence and this product induces a semigroup structure in the set of equivalence classes of $B_{N, M}^{\prime}$. We denote this semigroup by $B_{N, M}$. An element of $B_{N, M}$ is called a knit and the semigroup $B_{N, M}$ is called a knit semigroup.

Fig. 9

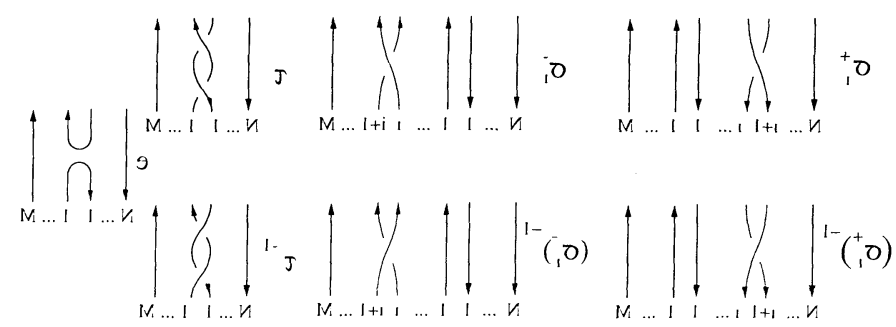

3.2. Algebra $H_{N, M}(a, q)$. The HOMFLY polynomial $P$ of links are defined recursively by the skein relation (1.3). The quotient of the group ring $\mathbf{C} B_{N}$ by the skein relation is the Iwahori-Hecke algebra and $P$ is given by a trace of it. Similarly, the quotient of the semigroup ring $\mathbf{C} B_{N, M}$ by the skein relation is the algebra $H_{N, M}(a, q)$ introduced in [Ko-M1, Ko-M2]. It is defined by the following generators and relations.

$$
\begin{aligned}
H_{N, M}(a, q)= & \left\langle T_{1}^{+}, \ldots, T_{N-1}^{+}, T_{1}^{-}, \ldots, T_{M-1}^{-}, E / T_{\imath}^{ \pm} T_{i+1}^{ \pm} T_{\imath}^{ \pm}=T_{i+1}^{ \pm} T_{i}^{ \pm} T_{\imath+1}^{ \pm}\right. \\
& T_{i}^{ \pm} T_{j}^{ \pm}=T_{j}^{ \pm} T_{i}^{ \pm}(|i-j| \geq 2), T_{\imath}^{ \pm} T_{j}^{\mp}=T_{j}^{\mp} T_{i}^{ \pm} \\
& E T_{i}^{ \pm}=T_{i}^{ \pm} E(i \geq 2), E\left(T_{1}^{+}\right)^{-1} T_{1}^{-} E T_{1}^{+}=E\left(T_{1}^{+}\right)^{-1} T_{1}^{-} E T_{1}^{-} \\
& E T_{1}^{ \pm} E=a^{-1} E, \quad E^{2}=-\frac{a-a^{-1}}{q-q^{-1}} E \\
& T_{1}^{+} E\left(T_{1}^{+}\right)^{-1} T_{1}^{-} E=T_{1}^{-} E\left(T_{1}^{+}\right)^{-1} T_{1}^{-} E \\
& \left.\left(T_{i}^{ \pm}-q\right)\left(T_{i}^{ \pm}+q^{-1}\right)=0\right\rangle
\end{aligned}
$$

The invariant $P$ of a link is expressed in terms of the Markov trace of this algebra. Let $p_{N, M}$ be an homomorphism from $\mathbf{C} B_{N, M}$ to $H_{N M}(a, q)$ defined by

$$
p_{N, M}\left(\sigma_{i}^{ \pm}\right)=a T_{i}^{ \pm}, \quad p_{N, M}(e)=E, \quad p_{N, M}(\tau)=-a^{-1}\left(q-q^{-1}\right) E+a^{-2}
$$

Let $b \in B_{N, M}$. By making a closure of $b$ as in the case of a braid, we get an oriented link diagram $\hat{b}$. Let $P_{N, M}(b)=P(\hat{b})$ for $b \in B_{N, M}$. Then $P_{N, M}$ is a composition of the mapping $p_{N, M}$ and a trace on $H_{N, M}(a, q)$.

3.3. Polytangle Diagram. Let $Q$ be a $n$-sided polygon. Let $\mathbf{s}^{(1)}=\left(s_{1}^{(1)}, s_{2}^{(1)}, \ldots\right)$, $\mathbf{s}^{(2)}, \ldots, \mathbf{s}^{(n)}$ be finite sequences of \pm 1 . We assume that the total number of elements 
of +1 in $\mathbf{s}^{(1)}, \mathbf{s}^{(2)}, \ldots, \mathbf{s}^{(n)}$ is equal to that of -1 in them. An $\left(\mathbf{s}^{(1)}, \mathbf{s}^{(2)}, \ldots, \mathbf{s}^{(n)}\right)$ polytangle $T$ is something like an oriented link diagram in $Q$. Some strings are non-closed and their end points are on the sides of $Q$. We give numbers to the sides of $Q$ anti-clockwise. Let $\left|\mathbf{s}^{(i)}\right|$ be the numbers of elements in $\mathbf{s}^{(i)}$. The $i^{\text {th }}$ side has $\left|\mathbf{s}^{(i)}\right|$ end points and the string intersecting with the $j^{\text {th }}$ point on the edge is oriented inwards if $s_{\jmath}^{(i)}=1$ and is oriented outwards if $s_{j}^{(i)}=-1$.

The skin relation (1.3) reduces every polytangle to a linear combination of some simple ones. A polytangle $T$ is called basic if it satisfies the following:

(1) There is no closed curve in $T$.

(2) Every string of $T$ has no self intersection.

(3) Any two strings of $T$ has at most one positive intersection.

The number of basic polytangles is equal to $r$ !, where $r=\sum_{i}\left|\mathbf{s}^{(i)}\right| / 2$.

3.4. Proposition. By using the skein relation (1.3), every polytangle is reduced to a linear combination of basic polytangles.

3.5. Colors of Edges and Vertices. Let $\Gamma$ be an oriented spacial graph. In what follows in this paper, a spacial graph means an oriented spacial graph. For $v \in \mathscr{T}_{\Gamma}$, let $\mathscr{E}_{v}$ be the set of edges with end point $v$. For $e \in \mathscr{E}_{\Gamma}$, we associate a pair $\left(N_{e}, M_{e}\right)$ of non-negative integers. We assume that

$$
\sum_{e \in \mathscr{E}_{v}} \varepsilon(e)\left(N_{e}-M_{e}\right)=0
$$

for every $v \in \mathscr{T}_{\Gamma}$, where $\varepsilon(e)=1$ if $e$ starts at $v, \varepsilon(e)=-1$ if $e$ ends at $v$, and $\varepsilon(e)=0$ if $e$ is a loop from $v$ to $v$.

Let $\widehat{H_{N, M}}(a, q)$ be the set of isomorphism classes of irreducible representations of $H_{N, M}(a, q)$. For an edge $e \in \mathscr{E}_{\Gamma}$, let $\phi(e) \in H_{N_{E}, M_{E}}(a, q)$. We call $\phi(e)$ the color of $e$. Let $\mathbf{s}_{e}$ be a sequence $(\underbrace{1,1, \ldots, 1}_{N_{e}}, \underbrace{-1, \ldots,-1}_{M_{e}})$. For $v \in \mathscr{T}_{\Gamma}$, let $n_{v}$ be the number of radii in $D_{v}$ associated to edges with an end point at $v$. Such radii in $D_{v}$ are denoted orderly by $r_{1}^{(v)}, r_{2}^{(v)}, \ldots, r_{n_{v}}^{(v)}$ and the corresponding edges are denoted by $e_{1}^{(v)}, e_{2}^{(v)}, \ldots, e_{n_{v}}^{(v)}$ respectively. Let $Q_{v}$ denote the set of basic polytangles of an $n_{v^{-}}$ sided polygon with sequences $\mathbf{s}_{e_{1}^{(v)}}, \mathbf{s}_{e_{2}^{(v)}}, \ldots, \mathbf{s}_{e_{n v}^{(v)}}$. Now we define colors of vertices. Let $\psi$ be a mapping from $\mathscr{T}_{\Gamma}$ to $Q_{v}$. For a vertex $v \in \mathscr{V}_{\Gamma}$, we call $\psi(v)$ the color of $v$.

3.6. Invariant of Embeddings. We define a regular isotopy invariant of embeddings of a special graph $\Gamma$ colored by $\phi$ and $\psi$ as above. For $\varrho \in \widehat{H_{N, M}}(a, q)$, let $\xi_{\varrho}$ be the ccorresponding central idempotent in $H_{N, M}(a, q)$. Let $\zeta_{\varrho}$ be an element of $\mathrm{C} B_{N, M}$ such that $p_{N, M}\left(\zeta_{\varrho}\right)=\xi_{\varrho}$. Note that $\zeta_{\varrho}$ is presented by a linear combination of diagrams. Let $G$ be a diagram of an embedding of $\Gamma$. Let $G_{\phi, \psi}$ be the linear combination of oriented link diagrams obtained by replacing edges and vertices of $G$ as in Fig. 10. The above replacing rule for a vertex $v$ is depend on the order of the edges $e_{1}^{(v)}, \ldots, e_{n_{v}}^{(v)}$ around $v$ in $G$. Let $P_{\phi, \psi}(G)=P\left(G_{\phi, \psi}\right)$. 


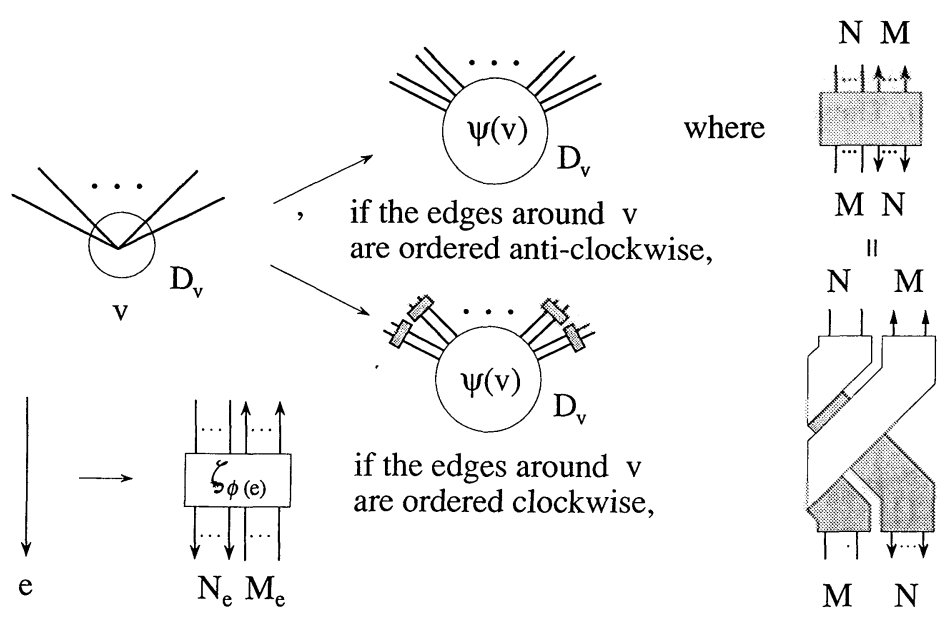

Fig. 10

3.7. Theorem. $P_{\phi, \psi}(G)$ is an invariant of regular isotopy.

Proof. The link represented by $G_{\phi, \psi}$ is invariant under the moves (RII), (RIII), and (RIV) in Fig. 6. Therefore, $P_{\phi, \psi}(G)$ is also invariant under these moves.

3.8. Full Twist. Let $t^{(N, M)}$ be a full twist given in Fig. 11. It is an element of the knit semigroup $B_{N, M}$ and commutes with all the elements of $B_{N, M}$. Therefore, irreducible representation $\varrho$ sends $p_{N, M}\left(t^{(N, M)}\right)$ to a scalar matrix.

Fig. 11

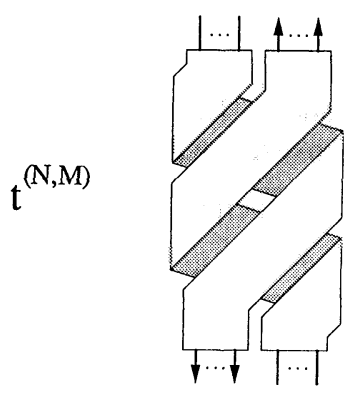

\subsection{Lemma.}

(1) $\varrho\left(p_{N, M}\left(t^{(N, M)}\right)\right)=\frac{\chi_{\varrho}\left(t^{(N, M)}\right)}{\chi_{\varrho}(1)} i d$.

(2) The scalar $\frac{\chi_{\varrho}\left(t^{(N, M)}\right)}{\chi_{\varrho}(1)}$ is a monomial in $a^{ \pm 1}$ and $q^{ \pm 1}$.

Proof. Part (1) is obvious because $\varrho\left(p_{N, M}\left(t^{(N, M)}\right)\right)$ is a scalar matrix. The determinant of $\varrho\left(p_{N, M}\left(t^{(N, M)}\right)\right)$ is also a monomial in $a$ and $q$ since the eigenvalues of $\varrho(E)$ 
and $\varrho\left(T_{i}^{ \pm}\right)$are monomials in $a$ and $q$. On the other hand, $\varrho\left(p_{N, M}\left(t^{(N, M)}\right)\right)$ can be represented by a matrix whose entries are rational functions on $a$ and $q$ (Theorem 4.12 of [Ko-M1]). These facts imply the part (2).

This lemma implies the following.

3.10. Lemma. The regular isotopy invariant $P_{\phi, \psi}$ satisfies the following relations:

$$
\left(\frac{\chi_{\phi(e)}\left(\mathrm{t}^{(\mathrm{M}, \mathrm{N})}\right)}{\chi_{\phi(\mathrm{e})}(1)}\right)^{-1} \mathrm{P}_{\phi, \psi}(\bigcirc)=\mathrm{P}_{\phi, \psi}(\bigcirc)=\frac{\chi_{\phi(\mathrm{e})}\left(\mathrm{t}^{(\mathrm{M}, \mathrm{N})}\right)}{\chi_{\phi(\mathrm{e})}(1)} \mathrm{P}_{\phi, \psi}(\bigcirc)
$$

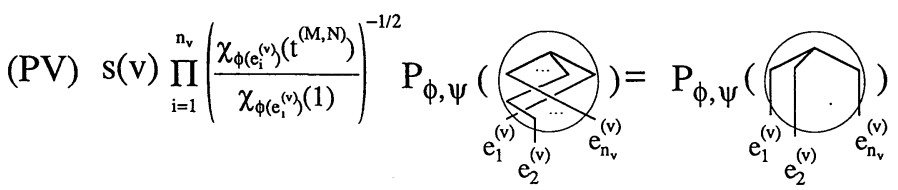

$$
=s(v) \prod_{i=1}^{n_{v}}\left(\frac{\chi_{\phi\left(e_{1}^{(v)}\right)}\left(t^{(M, N)}\right)}{\chi_{\phi\left(e_{i}^{(v)}\right)}(1)}\right)^{1 / 2} P_{\phi, \psi}(\underbrace{(v)}_{e_{1}^{(v)}}
$$

The following theorem is an immediate consequence of the above two lemmas.

3.11. Theorem. The regular isotopy invariant $P_{\phi, \psi}$ is an invariant of equivalence of embeddings of $\Gamma$ up to a multiple of a monomial in $a^{ \pm 1 / 2}$ and $q^{ \pm 1 / 2}$.

\section{Some Special Cases}

Let $\Gamma$ be a spacial graph and $\phi, \psi$ be colors of edges and vertices of $\Gamma$. We consider some special cases of coloring $\phi$ and $\psi$. Before doing it, we review the structure of the algebra $H_{N, M}(a, q)$, which is investigated (Theorem 4.12 of [Ko-M1]).

4.1. Structure of $H_{N, M}(a, q)$. The irreducible representations of $H_{N, M}(a, q)$ is parametrized by the set $\left.\Lambda_{N, M}=\{\lambda, \mu) \mid \lambda \in \Lambda_{N-k}, \mu \in \Lambda_{M-k}(k \geq 0)\right\}$, where $\Lambda_{k}$ is the set of partitions of size $k$. For $(\lambda, \mu) \in \Lambda_{N, M}$, let $\varrho_{\lambda, \mu}^{(N, M)}$ be the irreducible representation of $H_{N, M}(a, q)$ parametrized by $(\lambda, \mu)$ and $\chi_{\lambda, \mu}^{(N, M)}$ be its character. The construction of representations of $H_{N, M}(a, q)$ in [Ko-M1] implies the following.

4.2. Lemma. Let $H_{N, M}^{+}(a, q)$ be the two-sided ideal of $H_{N, M}(a, q)$ spanned by the simple components corresponding to $\chi_{\lambda, \mu}^{(N, M)}$ with $|\lambda|+|\mu|<N+M$.

(1) The subalgebra $H_{N, M}^{+}(a, q)$ is equal to the two-sided ideal generated by E.

(2) $H_{N, M}(a, q) / H_{N, M}^{+}(a, q) \cong H_{N, 0}(a, q) \otimes H_{0, M}(a, q)$.

4.3. Colored by Primitive Characters. An pair $(\lambda, \mu) \in \Lambda_{N, M}$ is called primitive if $|\lambda|+|\mu|=N+M$. The above lemma implies the following.

4.4. Lemma. Let $\xi_{\varrho_{\lambda, \mu}^{(N, M)}}$ be the central idempotent corresponding to $\varrho_{\lambda, \mu}^{(N, M)}$ as in 3.5. Then, for primitive $(\lambda, \mu) \in \Lambda_{N, M}$, we have $\xi_{\varrho_{\lambda, \mu}^{(N, M)}} E=E \xi_{\varrho_{\lambda, \mu}^{(N, M)}}=0$. 
The coloring $\phi$ is called primitive if $\phi(e)$ is primitive for every edge $e$. A basic polytangle $T$ is called primitive if it has no string whose terminal points are both on the same side of the polyhedron. The coloring $\psi$ is called primitive if $\psi(v)$ is basic and primitive for every vertex $v$. Then the above lemma implies the following.

4.5. Proposition. The invariant $P_{\phi, \psi}$ is zero for all embeddings of $\Gamma$ if $\phi$ is primitive and $\psi$ is not primitive.

4.6. Colored by Linear Characters. A character of $H_{N, M}(a, q)$ is called a linear character if the degree of the corresponding representation is 1 . The coloring $\phi$ of edges of $\Gamma$ is called linear if $\phi(e)$ is a linear character for every edge $e$. One dimensional representation sends every element of $H_{N, M}(a, q)$ to a scalar. Moreover, if the corresponding character is primitive, this representation sends $T_{i}^{+}$to $q$ or $-q^{-1}$, $T_{i}^{-}$to $q$ or $-q^{-1}$ and $E$ to 0 . This fact leads us to the following proposition.

4.7. Proposition. Let $\phi$ and $\psi$ are primitive colorings of edges and vertices of a spacial graph $\Gamma$. Assume that $\phi$ is linear. Let $\psi^{\prime}(v)$ be the tangle obtained by smoothing crossing points of $\psi(v)$ with two strings having the same starting side or the same ending side. Then $P_{\phi, \psi}$ is a multiple of $P_{\phi, \psi^{\prime}}$ by a monomial in $q$.

4.8. Orientation Free Case. Let $\Gamma$ be a spacial graph and $\phi, \psi$ be colorings of edges and vertices of it. Let $e$ be an edge of $\Gamma$ and $\phi(e)=(\lambda, \mu) \in \Lambda_{N_{e}, M_{e}}$. The invariant $P_{\phi, \psi}$ is called orientation free at the edge $e$ if $N_{e}=M_{e}$ and $\lambda=\mu$, since $P_{\phi, \psi}$ does not depend on the orientation of $e$. The invariant $P_{\phi, \psi}$ is called orientation free if it is orientation free at all the edges. In this case, $P_{\phi, \psi}$ is an invariant of embeddings of a non-oriented spacial graph.

4.9. Symmetric Case. Let $\phi$ and $\psi$ be primitive colorings of edges and vertices of a spacial graph $\Gamma$. Assume that $\phi$ is orientation free and a constant mapping. Let $n_{v}$ be the degree of a vertex $v$. Let $\mathscr{D}_{2 n_{v}}$ be the dihedral group with $2 n_{v}$ elements. For $g \in \mathscr{D}_{2 n_{v}}$, we define $g \cdot \psi(v)$ as follows. If $g$ is a rotation, $g \cdot \psi(v)$ is a rotation of $\psi(v)$. If $g$ is a reflection, $g \cdot \psi(v)$ is a flip of $\psi(v)$ added by half twist as in Fig. 12. Let $\mathbf{C} D_{v}$ denote the set of formal linear combinations of elements in $D_{v}$. We extend linearly the definition of $P_{\phi, \psi}$ in case that $\psi$ is a mapping with values in $\mathbf{C} D_{v}$. The coloring $\psi$ is called symmetric at a vertex $v$ if $g \cdot \psi(v)$ is a scalar multiple of $\psi(v)$. The coloring $\psi$ is called symmetric if $\psi$ is symmetric at all the vertices.

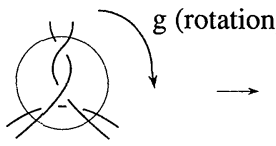

$\psi(\mathrm{v})$

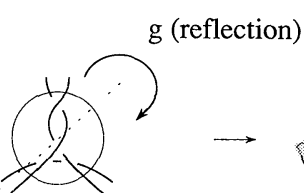

Fig. 12

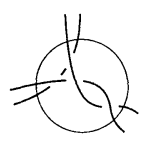

$\mathrm{g} \cdot \psi(\mathrm{v})$

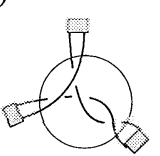

where
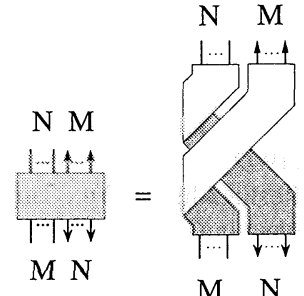

$\mathrm{g} \cdot \psi(\mathrm{v})$ 
The invariant $Z_{s}$ in Sect. 1 is equal to $P_{\phi, \psi}$ with colorings $\phi$ and $\psi$, where $N_{e}=M_{e}=1, \phi(e)=((1),(1)) \in \Lambda_{1,1}$ for each edge $e$ and $\psi(v)$ is the linear combination of polytangles in Fig. 4 for each vertex $v$.

4.10. Proposition. The invariant $Z_{s}$ is primitive, linear, and symmetric.

4.11. Trivalent Case. Let $\Gamma$ be a trivalent spacial graph, i.e. the degree of every vertex is equal to three. Let $\mathscr{C}_{s}$ be the category of embeddings of $\Gamma$ as a spacial graph and let $\mathscr{C}_{a}$ be that as an abstract graph. Then there is a natural isomorphism between $\mathscr{C}_{s}$ and $\mathscr{C}_{a}$. Therefore, Theorem 3.11 implies the following.

4.12. Corollary. $P_{\phi, \psi}$ is an ambient isotopy invariant of embeddings of a trivalent graph colored by $\phi$ and $\psi$ up to a multiple of a monomial in $a^{ \pm 1 / 2}$ and $q^{ \pm 1 / 2}$.

\section{Discussion}

Our invariant $P_{\phi, \psi}$ has two parameters $a$ and $q$. If we specialize $a=q^{-n}$ for an integer $n \geq 2$, this invariant is essentially equal to that in [R-T] in the case $\mathscr{A}=\mathscr{Q}_{q}\left(g l_{n}\right)$ with coloring corresponding to $\phi$ and $\psi$. While they associate a simple $\mathscr{C}_{q}\left(g l_{n}\right)$-module to an edge in [R-T], we associate a simple $H_{N, M}(a, q)$ module to an edge. These two simple modules are related as follows. Let $V$ denote the vector representation of $\mathscr{C}_{q}\left(g l_{n}\right)$ and $V^{*}$ be its contra-gradient representation. Then simple $H_{N, M}(a, q)$-modules parametrize simple components of the mixed tensor representation $V^{\otimes N} \otimes\left(V^{*}\right)^{\otimes M}$ if $n$ is sufficiently large. Therefore, our parametrization corresponds to that in [R-T]. However, our method does not depend on $n$. As for vertices, we give a specific method to determine the coloring of vertices by using polytangles.

We can replace $H_{N, M}(a, q)$ by a braided version of a rigid category generated by a single object. The results of this paper may hold in this generalized case.

\section{References}

[FY-H-LM-O] Freyd, P., Yetter, D., Hoste, J., Lickorish, W.B.R., Millett, K.C., Ocneanu, A.: A new polynomial invariant of knots and links. Bull. Am. Math. Soc. 12, 239-245 (1985)

[Ja] Jaeger, F.: On some graph invariants related to the Kauffman polynomial. Preprint 1989

[Jo] Jones, V.F.R.: A polynomial invariant for knots via von Neumann algebras. Bull. Am. Math. Soc. 12, 103-111 (1985)

[Ka1] Kauffman, L.H.: State models and the Jones polynomial. Topology 26, 395-407 (1987)

[Ka2] Kauffman, L.H.: An invariant of regular isotopy. Trans. Am. Math. Soc. 318, 417-471 (1990)

[Ko-M1] Kosuda, M., Murakami, J.: Centralizer algebras of the mixed tensor representations of quantum algebra $\mathscr{2} b_{q}\left(g l_{n}\right)$. To appear in Osaka J. Math.

[Ko-M2] Kosuda, M., Murakami, J.: The centralizer algebras of mixed tensor representations of $\mathscr{Q}_{q}\left(g l_{n}\right)$ and the HOMFLY polynomial of links. Proc. Jpn. Acad. Ser. A 68, 148-151 (1992)

[R-T] Reshetikhin, N.Yu., Turaev, V.G.: Ribbon graphs and their invariants derived from quantum groups. Commun. Math. Phys. 127, 1-26 (1990)

[Y] Yamada, S.: An invariant of spacial graphs. J. Graph. Theory 13, 537-551 (1989) 